\title{
Tiwouh: an enriched technology-based solution for communication interventions
}

\author{
${ }^{*}$ C. Fage, ${ }^{*}$ S. Giblet, $* *$ M. Jaspard, ${ }^{* * * P}$. Grevesse, ${ }^{*}$ C. Maillart \\ *Unité de Logopédie Clinique, Faculté de Psychologie, Logopédie et Sciences de l'Éducation, \\ Université de Liège \\ Belgique, rue de l'Aunaie, 30, Angleur \\ (charles.fage@uliege.be,sarah.giblet@,student.uliege.be,christelle.maillart@uliege.be) \\ ** Centre d'Accueil de jour La Fermette, Services de l'APEM \\ Belgique, Verviers (m.jaspard@servicesapem-t21.eu) \\ *** Orthophoniste indépendante \\ Belgique, Obaix (pgrevesse@hotmail.be)
}

\begin{abstract}
Democratizing mobile technological devices lead to the development and spreading of tools supporting communication of individuals with Intellectual Disabilities (ID). Although promising, these tools exhibit limited impacts in daily life given their limited features and the lack of health professionals support. This article presents Tiwouh, an enriched technological solution to support communication interventions. After documenting the needs, this solution has been deployed and used in real life situations within a daycare facility for adults with ID.
\end{abstract}

\section{Key words}

AAC, Intellectual Disability, Assistive Technology, Field Study, Daily-life activities

\section{Introduction}

Mobile technologies have rapidly spread to every aspect of our lives, and particularly for individuals with Intellectual Disabilities (ID). These individuals exhibit limited abilities in dailylife activities, greatly impeding their social participation [1]. Technological devices, especially when they are mobile, offer opportunities to support individuals in situ, in their daily lives. These 
tools have been developed to support individuals with ID mainly in their communication [2], as well as their activities (initiating, transitioning, etc.) [3].

\subsection{Assistive Technologies for Individuals with ID}

\subsubsection{Alternative and Augmentative Communication (AAC) Supports}

AAC refers to strategies and tools an individual can use to cope with daily-life communication challenges (International Society of AAC). The most commonly used AAC method - and the most documented for individuals with ID - is certainly the Picture Exchange Communication System (PECS, [4]). This strongly structured behaviorist method (i.e. divided in successive learning steps with success criteria) is based on the exchange of paper-based pictures with a communication partner; it has been reported successful for individuals with ID [5,6]. More recently, leveraging opportunities offered by mobile devices (tablets, smartphones), assistive tools have been developed to complement the use of pictures/pictograms with synthetic speech (e.g. MyVoice application [7]). When a pictogram is selected, the synthetic speech produces the matching sound. Such tool allows users to produce oral language (mechanically) and be exposed to oral models easing learning words used in their messages.

In a recent meta-analysis of these interventions towards individuals with ID, authors reported their efficacy to improve communication of this population [8]. Authors also argued that such good results can be observed in protected environments but also in mainstream environments, therefore suggesting that providing support in situ may promote generalization of learnings. Moreover, improving communication can lead to a decrease in challenging behaviors, since these behaviors are often linked with frustration when individuals cannot express their needs [9].

\subsubsection{Activity Schedules}

Activity schedules are based on picture and/or text sequences decomposing an activity into successive steps. Classically used on their paper form, they have been implemented on mobile devices to support individuals with ID in transitions between activities [3], and even directly in school inclusion [10]. After documenting the needs, authors successfully proposed activity schedules to support the communication and activities of students with ID in mainstream classrooms. Previous reviews also revealed promising results from interventions based on mobile devices to improve communication and daily-living skills of this population $[11,14]$.

\subsection{Limits of Existing Solutions}


Although promising, interventions based on AAC tools and activity schedules on mobile devices present several limitations reducing the impact of reported results. First, authors highlight a lack of generalization of benefits on novel contents or environments [9]. In the same vein, the lack of measures related to social validity (only 2 on 15 reviewed studies) questions both the feasibility and relevance of such interventions in real-life situations [9]. Moreover, literature mostly investigated the effects of these interventions on children with ID, and data addressing adults with ID are very limited [8].

Furthermore, several authors pinpointed that communication interventions focus almost exclusively on developing basic requests skills, and urge to go beyond to develop other communication aspects $[8,9]$. In that sense, AAC tools often offer "basic" features only allowing selecting a pictogram and its vocalization [11,12]. However, technological tools offer opportunities that can go beyond these features and therefore enrich communication interventions.

Finally, the large spreading of these assistive technologies among individuals with disabilities urges for supervision or coaching by trained health professionals (speech-language pathologist, typically) to support adequate usages. Users are too often on their own during the choice, the use and the purpose of an AAC tool $[11,12]$.

\subsection{Contributions}

In this context, the Tiwouh project proposes a technological solution to support AAC interventions. It is deployed on the field and used by individuals with various conditions who exhibit complex communication needs - mainly Autism Spectrum Disorders and Intellectual Disabilities) in French-speaking regions of Belgium (CMAP Bruxelles), France (IME Les Hautes-Roches, Saint-Malo), but also in Lebanon, Switzerland and Quebec. This paper presents the Tiwouh solution with an example of our approach based on the needs for a deployment within a daycare facility for adults with ID.

\section{An Enriched AAC Technological Solution}

The Tiwouh solution, based on the needs of speech-language pathologists (SLPs) and developed by a pluri-disciplinary team (SLPs, experts in Human-Computer Interface for disability, developers), is based on both an online plate-form and a mobile application. This architecture can improve classical AAC interventions, especially by bringing stakeholders 
together around the patient. Moreover, a systematic scientific validation is conducted for each population and intervention domain where the solution is deployed.

\subsection{Design and Functional Validation}

Tiwouh is based on the needs of SLPs in terms of mobile technological supports for interventions. 19 SLPs participated in focus groups to identify categories of needs regarding contents, interface and features. Among them, the most critical needs were related to the diversity of domains to be supported as well as individualization and adjusting complexity levels [12].

Following this work, prototypes of both an online plate-form and a mobile application have been developed; the first version of the tool was tested by several independent SLPs. These early tests lead to adjustments on contents and interface to ensure functional validation of the tool. Finally, a stabilized version was released to various beta-testers (e.g. SLPs in specialized institutions, parents, psychologists, special-education teachers, etc.) in order to validate the scaling of the plate-form. This last step confirmed the relevance of the chosen architecture.

\subsection{Architecture and Functioning}

Tiwouh solution is based on an online plate-form and a mobile application. The plate-form is a website taking the form of a workshop in which users (stakeholder, typically) will be able to manage communication boards: creation, modification, personalization, etc. All contents can be entirely modified, from pictograms to pronunciation; new images can also be uploaded to the plate-form. This is also the place where the user will manage his/her patient group: separate and individualized profiles can be created for any given account. This feature particularly suits structures by addressing the different needs of their patients through one single solution.

Mobile application is connected with the plate-form with wi-fi for profiles and contents to be downloaded; it can be used offline in daily-life afterwards. Additional to full content individualization in the plate-form, the application presents various parameters related to its overall functioning: voice choice, displaying texts with pictograms, content size, etc. but also related to each feature specifically (e.g. randomly displaying pictograms, reinforcer type).

\subsection{Extended Features}

As almost every existing AAC tool, the first feature of the Tiwouh solution is the PECS-like communication board: pictograms are organized in a grid, above which a sentence strip allows selected pictograms to be vocalized sequentially (see Figure 1). However, in order to address 
limits identified in the literature review above, Tiwouh also integrates several other features, originating either from paper-based tools already used in the field with individuals with ID (e.g. activity schedules) or existing method used in clinical communication interventions. Extended features are presented below.

Sequences in Tiwouh are equivalent to activity schedules. This feature allows creating successive steps, each illustrated by a pictogram/picture. A checkbox is also displayed on each step: if each one is validated, the reinforcer is displayed at the end of the sequence. Including this feature in communication interventions has been suggested in the literature, given the positive impact of its use on other interventions [14].

Contents of the Vocabulary feature present the user different possible answers to an instruction written and synthetically vocalized. A wrong answer will lead to no reaction from the interface: only the text of the selected pictogram will be changed into red mentioning it has already been selected. A good answer generates a new proposition, and possible answers will again be randomly displayed. The presence of a reinforcer can be chosen directly within the parameters of the application, as for the number of tests for each instruction, etc.

The Description feature allows decomposing a given picture into its different elements. For example, for the picture of a dog sleeping, the feature will propose three distinct elements: a dog, a color and a person sleeping. The user is to drag-and-drop the adequate pictograms in corresponding places to recreate the picture description.

In the Spelling feature, the target picture is displayed and vocalized at the beginning of the exercise: the word is written in plain letters on the top of the screen and corresponding letters are randomly displayed below.

The Pointing feature randomly displays a pictogram/picture in a grid. Each time the user successful selects the target, it is immediately randomly positioned elsewhere on the grid.

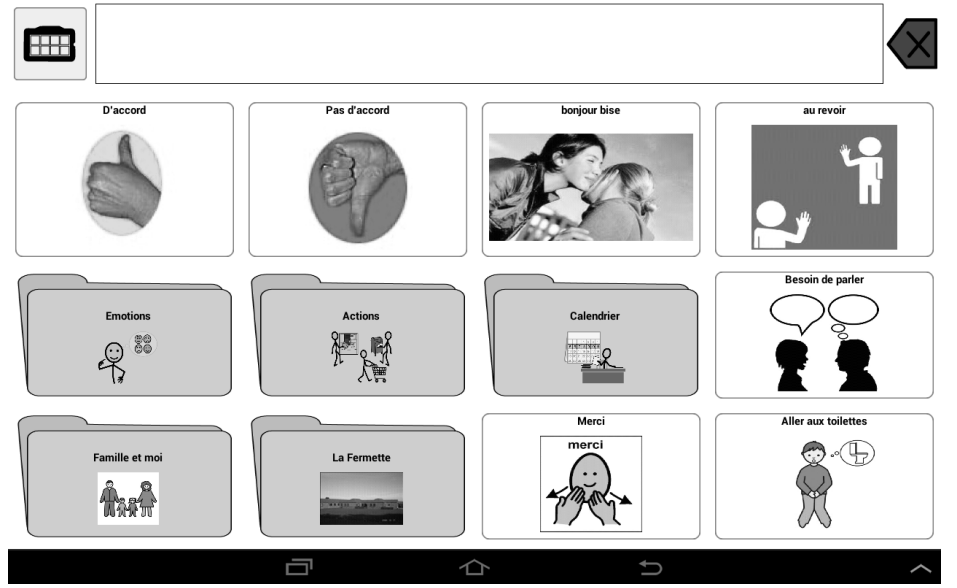

Fig.1. Example of Communication Board in Tiwouh Application 


\subsection{Building a Community to Bring Stakeholders Together}

The Tiwouh solution has been designed around an online plate-form in order to bring stakeholders together. Parents of individuals with ID are often isolated and lack information or resources to cope with everyday challenges. To address this issue, a functionality has been added in Tiwouh where a parent-account can be linked with a patient whose profile has been created by his/her health professional. Therefore, the professional will be able to share the patient's contents used in the intervention directly to his/her parents, bringing the intervention at home.

More broadly, contents are already available within the plate-form: they can be downloaded at any time and modified to fit the specific needs of everyone. Users themselves can propose contents they created to be shared with the community, with SLPs from the Tiwouh staff to ensure the content quality.

Finally, information materials are being elaborated in order to support users on their knowledge towards interventions that can be implemented with the tool. The different deployments of the solution already achieved allow us to develop detailed contents adapted to different contexts and populations. A forum is also linked with the plate-form allowing users to communicate directly with each other with the privacy of a dedicated community.

\section{Need-Based Approach}

Since its genesis, Tiwouh strongly relies on identifying the needs to be the closest to the users' expectations. This approach is also adopted for every collaboration and deployment on the field: the team gathers as much information as possible on the expectations and constraints in order to benefit the most for each specific context addressed and therefore ensure the adoption of the solution.

\subsection{Presentation of Daycare “La Fermette” Structure}

This structure is a daycare facility for adults with ID ranging from moderate to severe disability. Communication skills of the adults are variable: some have fairly good abilities in understanding by extremely limited in production. Some adults used mobile-based AAC tools. However, without speech-language training, these tools have limited impact, particularly through the diversity of contexts within the facility.

La Fermette is a living and working place, where adults can participate in various activities such as sports, wellbeing, dynamization and also leisure. Different activities are proposed to them: outside (firewood, pedagogical farm), catering, etc. The staff is constituted by educators, an 
Occupational Therapist (OT) and a psychologist. An SLP can be contacted in specific cases, but this represents very limited resources for communication interventions. This issue led the structure to contact our clinical-research team to be supervised in properly adapting and using an AAC tool.

\subsection{Diversity of Needs}

We conducted interviews with La Fermette staff that allowed us to identify specific needs but also diverse regarding a Tiwouh-based intervention. Unsurprisingly, these needs perfectly match the limits in the literature identified in the Introduction section of this paper.

\subsubsection{Allowing adults to express themselves and to be understood}

The first clear need we identified was related to the difficulties the staff faced in understanding the adults. Although highly motivated to communicate, adults struggle to be understood. This results in tremendous frustration for the adults, but also for the staff members. Educators have strong time constraints throughout the day and have limited time for unformal interactions that might take longer than usual. Giving adults means to express themselves more clearly appears mandatory, as well as training staff members to improve their understanding.

\subsubsection{Disseminating the solution amongst La Fermette staff}

Echoing the first point, the supervising team of La Fermette expressed their wish to specifically address the solution dissemination amongst staff members. A sign-based AAC intervention (i.e. based on the SESAME method) had previous been implemented within the facility, and the biggest barrier was related to staff members being reluctant to learn the signs and how to properly use them in situation. Consequently, the intervention was stopped, leaving the adults without communication support for a long period of time. This finding is consistent with the clinical action-research literature: resisting change and structural constraints have to be addressed in the earliest phases of a deployment to favor adoption of a new tool [10].

\subsubsection{Supervising tool usage through different contexts}

The third crucial identified need was related to the diversity of contexts in which adults with ID need communication supports. They participate in different activities throughout the day, and benefit from specialized interventions (OT, psychology) and sometimes go back home autonomously. Thus, any tool aiming to support their communication should address these 
different contexts, and the intervention should favor its usage through them. Moreover, benefits from deploying assistive technologies on natural settings have recently been reported [8].

Globally, documenting these needs highlights the importance of broadly spreading pedagogical contents addressing communication interventions, and particularly in the AAC domain given the variety of available tools. Similarly, limited resources of specialized structures regarding these communication interventions strengthen the necessity of supervision by trained personnel.

\section{In Situ Deployment}

Following the identification of such diverse needs, an SLP student has been recruited to implement a Tiwouh-based intervention three days a week during four months within the daycare facility. This intervention was implemented in three phases: content creation, staff members training, and adults support throughout the different contexts.

\subsection{Creating Contents}

All contents required for this intervention were created in close collaboration with all professionals of La Fermette. Notably, they indicated the communication abilities of the participants, their specific interests, and also took pictures needed for some communication boards (e.g. activities, staff members' faces, etc.). Therefore, every picture has been identified and uploaded on the plate-form and organized on the appropriate folders in order to be available for every content to be created afterwards. Three types of contents have been developed.

Several communication boards have been created for each participant of the intervention. Different domains have been targeted, depending on abilities and activities of everyone: daily activities (e.g. dressing, drinking), requests (e.g. going to bathroom), greetings, and emotions. Moreover, a personal communication board was created for each participant, in direct collaboration with him/her, and the family when required (family pictures, leisure, friends, etc.).

Sequences have been particularly used within the catering activity for the adults to autonomously follow recipes. This feature appears of great interest in this scenario given the time-consuming work conducted by the OT preparing the paper-based supports for this activity: printing pictures, cutting, laminating, etc. Plus, the mobile device seems less cumbersome than paper folders in the kitchens. The vocalization of each step instruction is particularly appreciated within this context. 
Other features available in Tiwouh were harnessed. Notably, adults could be trained through the Vocabulary feature on the lexicon involved in each activity they participated in. Some paperbased games have also been adapted to communication board to support adults' participation.

\subsection{Training Staff Members}

Following the content creation, staff members were presented with the Tiwouh solution and trained to use it. Individual interviews were conducted to highlight its relevance and how to use it properly. To do so, interviews were divided into three steps. On the first step, the staff member could freely explore the application to get familiar with it. On the second, participant asked and answered questions about the functioning of the tool; he/she had to retrieve information in order to assess his/her management of the tool. Finally, the third step involved the staff member actually using the tool in a role game. The staff member had to give instructions to the interventionist through the application. This procedure allowed staff members to master the tool usage, modifying potential false beliefs to favor its dissemination.

\subsection{Supporting Adult Communication}

The last phase of our intervention was related to actually supporting adults' communication throughout their activities in the daycare facility. Educators, although trained to use it, did not necessarily know how to integrate it into the activity they supervised. This work aimed at finding appropriate usages, in collaboration with the educator. This way, educators were able to perceive the relevance of the tool and how it could be integrated to their practice and adults understood that educators are also capable in using it to support them. Consequently, the technological solution was not associated with one dedicated person but with the entire pluri-disciplinary team.

\section{Conclusion / Future Work}

In this paper, we presented Tiwouh, a technological solution to support communication interventions in daily-life. This solution, deployed on the field, offers extended features to overcome the multiple challenges that individuals with complex communication needs may face, especially those with ID $[12,13]$.

We presented the deployment of the solution in a daycare facility for adults with ID. After identifying the needs and constraints of adults but also the structure, we developed adapted contents by involving as much staff members and adults as possible in order to address false beliefs and create cohesion to favor the tool dissemination. The presence of a referent person - in 
the form of an SLP student - was crucial for the deployment success. Capitalizing on previous attempts in this structure, this need had been identified from the very beginning by La Fermette supervision staff.

Following this deployment, several perspectives can be drawn. First, based on adults mastering the tool usage, health professionals can develop new activities to address other domains of communication, such as expressing emotions or telling stories. Second, thanks to the sharing content feature, participants' families now consider getting the solution to use the personal communication boards developed in this intervention [8]. To go further, we will develop training supports to allow users to develop their knowledge about AAC interventions. These supports will be diffused on the plate-form under different forms: videos, grids, datasheets, etc. Finally, even though the presence of a referent person was part of the success of this work, it is not sustainable over time. Remote coaching modalities will be investigated to keep supervising user and improving communication interventions.

\section{Acknowledgements}

Authors would like to give special thanks to every member of La Fermette staff who have put a lot of energy in this novel intervention. Adults have also been really helpful to implement this intervention. Finally, we thank Gaelle Liégeois for her precious support on communication interventions in La Fermette.

\section{References}

1. S., Mouga, J., Almeida, C., Café, F., Duque, \& G. Oliveira, “Adaptive profiles in autism and other neurodevelopmental disorders", Journal of autism and developmental disorders, vol. 45, $\mathrm{N}^{\circ} 4$, pp.1001-1012, 2015.

2. K., Nepo, M., Tincani, S., Axelrod, \& L. Meszaros, "iPod touch ${ }^{\circledR}$ to increase functional communication of adults with autism spectrum disorder and significant intellectual disability", Focus on Autism and Other Developmental Disabilities, vol. 32, № 3, pp.209$217,2017$.

3. D. F., Cihak, K., Kessler, \& P. A., Alberto, "Use of a handheld prompting system to transition independently through vocational tasks for students with moderate and severe intellectual disabilities", Education and Training in Developmental Disabilities, pp. 102-110, 2008.

4. L. A., Frost, \& A. S. Bondy, (1994). PECS training manual. Newark: Pyramid Educational Consultants 
5. M., Tincani, \& E. R. Lorah, "The Picture-Exchange Communication System (PECS) increases functional communication of adults with intellectual disabilities", Evidence-Based Communication Assessment and Intervention, vol. 5, $N^{\circ}$ 3, pp. 168-170, 2011.

6. B., Sulzer-Azaroff, A. O., Hoffman, C. B., Horton, A., Bondy, \& L. Frost, "The Picture Exchange Communication System (PECS) What Do the Data Say?", Focus on Autism and Other Developmental Disabilities, vol. 24, N², 89-103, 2009.

7. R., Campigotto, R., McEwen, \& C. D. Epp, "Especially social: Exploring the use of an iOS application in special needs classrooms", Computers \& Education, vol. 60, $\mathrm{N}^{\circ} 1$, pp. 74-86, 2013.

8. J. B., Ganz, K. L., Morin, M. J., Foster, K. J., Vannest, D., Genç Tosun, E. V., Gregori, \& S. L. Gerow, "High-technology augmentative and alternative communication for individuals with intellectual and developmental disabilities and complex communication needs: a metaanalysis", Augmentative and Alternative Communication, vol. 33, N 4, pp. 224-238, 2017.

9. N., Alzrayer, D. R., Banda, \& R. K. Koul, "Use of iPad/iPods with individuals with autism and other developmental disabilities: A meta-analysis of communication interventions", Review Journal of Autism and Developmental Disorders, vol. 1, $\mathrm{N}^{\circ}$ 3, pp. 179-191, 2014.

10. C., Fage, L., Pommereau, C., Consel, E., Balland, \& H. Sauzéon, "Tablet-based activity schedule in mainstream environment for children with autism and children with ID". ACM Transactions on Accessible Computing (TACCESS), vol. 8, N³/9, 2016.

11. D. M., Kagohara, L., van der Meer, S., Ramdoss, M. F., O’Reilly, G. E., Lancioni, T. N., Davis, ... \& V. A. Green, "Using iPods ${ }^{\circledR}$ and iPads ${ }^{\circledR}$ in teaching programs for individuals with developmental disabilities: A systematic review", Research in developmental disabilities, vol. 34, $\mathrm{N}^{\circ}$ 1, pp. 147-156, 2013.

12. D., McNaughton, \& J. Light, "The iPad and mobile technology revolution: Benefits and challenges for individuals who require augmentative and alternative communication", Augmentative and Alternative Communication, vol. 29, $\mathrm{N}^{\circ}$ 2, pp. 107-116, 2013.

13. C., Maillart, P., Grevesse, \& T. Martinez Perez, "Qu'attendent les orthophonistes des applications numériques à destination des personnes avec trouble $\mathrm{du}$ spectre autistique?", Rééducation Orthophonique, № 264, pp. 139-150, 2015.

14. J., Stephenson, \& L. Limbrick, "A review of the use of touch-screen mobile devices by people with developmental disabilities", Journal of autism and developmental disorders, vol. 45, $\mathrm{N}^{\circ} 12$, pp. 3777-3791, 2015. 